\title{
A NEW CAR-FOLLOWING MODEL CONSIDERING ACCELERATION OF LEAD VEHICLE
}

\author{
Bingrong Sun ${ }^{1}, \mathrm{Na} \mathrm{Wu}^{2}$, Ying-En $\mathrm{Ge}^{3}$, Taewan Kim${ }^{4}$, Hongjun Michael Zhang ${ }^{5}$ \\ ${ }^{1,2,3}$ School of Transportation and Logistics, Dalian University of Technology, Liaoning, China \\ ${ }^{3}$ College of Transport and Communications, Shanghai Maritime University, China \\ ${ }^{4}$ Dept of Urban Engineering, Chung-Ang University, Korea \\ ${ }^{5}$ Dept of Civil and Environmental Engineering, University of California, Davis, USA
}

Submitted 2 November 2013; accepted 20 August 2013; first published online 28 May 2014

\begin{abstract}
For decades, the General Motors (GM) car following model has received a great deal of attention and provided a basic framework to describe the interactions between vehicles on the road. It is based on the stimulus-response assumption that the following vehicle responds to the relative speed between the lead vehicle and itself. However, some of the empirical findings show that the assumption of GM model is not always true and need some modification. For example, the acceleration of the following vehicle is very sensitive to the sign of the relative speed and because of no term in the model that directly represents the leader's acceleration, the follower's response to the leader's acceleration can be retarded. This paper offers a new car-following model that can be considered as a variant of the GM model that can better capture car following behavior. The new model treats the follower's acceleration as a proportion of a weighted sum of the leader's acceleration and the relative speed between the lead and following vehicles. This paper compares the new model with the original GM model numerically and the characteristics of the new parameters in the model are investigated. It is also shown that the new model overcomes the shortcomings of the original GM model identified in this paper and gives us more instruments to capture the real-world car-following behavior.
\end{abstract}

Keywords: car-following behavior; microscopic traffic flow models; GM model; sensitivity; stability.

\section{Introduction}

The car-following behavior is one of the most common traffic phenomena on the road. A number of car-following models have been developed to capture the behavior of how a following vehicle or follower adjusts its own speed when the speed and position of the lead vehicle or leader - immediate front one - change.

The following General Motors (GM) model proposed in Gazis et al. (1961) gives the acceleration of vehicle $n+1$ following vehicle $n$ :

$$
\begin{aligned}
& \ddot{x}_{n+1}(t+\delta)=\alpha(l, m) \frac{\left(\dot{x}_{n+1}(t+\delta)\right)^{m}}{\left(x_{n}(t)-x_{n+1}(t)\right)^{l}} \times \\
& \left(\dot{x}_{n}(t)-\dot{x}_{n+1}(t)\right),
\end{aligned}
$$

where: $x_{n}(t)$ is the position of the $n$th car; $\dot{x}_{n}(t)$ and $\ddot{x}_{n+1}(t)$ respectively represent the speed and acceleration/deceleration of the $n$th vehicle at time $t ; \delta$ is the time lag of the follower's response to the stimuli (e.g. accelerating or decelerating) from the lead vehicle; $l$ and $m$ are parameters to be calibrated; $\alpha(l, m)$ is a positive constant whose value depends on the two parameters $l$ and $m$. In this model, the follower's acceleration at time $t+\delta$ monotonically increases with respect to (w.r.t.) its own speed at the time whereas monotonically decreases w.r.t. the distance headway between the two vehicles at time $t$ and is also proportional to their speed difference. All terms before the speed difference is usually known as the sensitivity coefficient and denoted as:

$$
\lambda=\alpha \frac{\left(\dot{x}_{n+1}(t+\delta)\right)^{m}}{\left(x_{n}(t)-x_{n+1}(t)\right)^{l}} .
$$

Therefore, model (1) can be written as the earliest form of the GM model (Chandler et al. 1958):

$$
\ddot{x}_{n+1}(t+\delta)=\lambda\left(\dot{x}_{n}(t)-\dot{x}_{n+1}(t)\right),
$$

which is known as a linear car-following model in Farhi (2012). Gazis et al. (1959) and Herman et al. (1959) re- 
spectively investigated the connection between the model and the flow-density relationship proposed in Greenberg (1959) and the stability of this model. The reason why the above models are called GM model is that they were developed in the General Motors' laboratory (Gazis 2002). Until now the findings given in Chandler et al. (1958) are still the footstone of microscopic traffic flow models. A great deal of research has been performed on the calibration and validation of the GM model (Brackstone, McDonald 1999). Among those the most notable are May and Keller (1967), Heyes and Ashworth (1972), Ceder and May (1976), who presented the combination of $l$ and $m$ values for a single state of traffic. In addition, Treiterer and Myers (1974) and Ozaki (1993) presented different combinations of $l$ and $m$ values for different states of traffic, such as acceleration, steady state, and deceleration. Despite the enormous efforts to obtain optimal values of $l$ and $m$, the empirical findings still deviate from the GM model because the car following behavior varies for different traffic conditions and the optimal values of $l$ and $m$ obtained from empirical data represent averages for various drivers.

On another hand, a great deal of literature has been contributed to the improvement of the GM model or investigation of many other aspects of the car following behavior, such as Lee (1966), Gazis (1967), Tolle (1974), Zhang and Jarrett (1997), Chakroborty and Kikuchi (1999), Aw et al. (2002), Nagel et al. (2003), Simões et al. (2010), Tordeux et al. (2010), Ge et al. (2011), Ge and Sun (2012). Subramanian (1996) found that drivers react faster in the deceleration phase than in the acceleration phase and Ahmed (1999) assumed that drivers behave differently in car-following and free flow states and improved Subramanian's model by adding traffic density in the sensitivity term. Siuhi and Kaseko (2010) argued that the GM family models have three limitations: (1) identical reaction times for all drivers; (2) a single value for each of the model parameters; (3) drivers are able to perceive even small stimulus. One of the key assumptions of the GM model is that the follower must decelerate when the leader's speed is smaller than the follower's, and that the follower must accelerate when the speed of the lead vehicle is larger than the follower's. However, the recently-available microscopic vehicle trajectory data (e.g. Next Generation SIMulation - NGSIM data) show that this is not always true and needs some modifications (Alexiadis et al. 2004). Koutsopoulos and Farah (2012) gave a flexible framework for modelling the car-following behavior that relaxes some limitations and assumptions of the GM model and showed that the latent class model performs better than the previous ones. As discussed above, while the GM car following model has provided a basic framework for most car following models and has been improved continuously in the past six decades, it still falls short in describing various car following behaviors and for certain traffic conditions. Therefore, the basic assumptions of the GM model need further relaxation. This paper intends to improve the GM model by incorporating some car following fea- tures that have not been captured by the basic premise or existing variants of the GM model.

The car-following model (1) considers the effects on the follower's acceleration of three types of factors, i.e. speed difference, gap and follower's speed. Let us look at a special but common phenomenon: the relative speed between the lead and following vehicles is zero and the acceleration of the leader is nonzero, where the follower's acceleration is nonzero. Under the circumstances the follower's acceleration given by model (1) is always zero; it should be noted that the response time is already embedded in model (1). However, in real-life traffic like this, the follower's acceleration is usually nonzero and the two vehicles might have the same or similar accelerations.

In addition, since there is no term in the model (1) that directly represents the leader's acceleration, the follower's response to the leader's acceleration is retarded, and it takes the follower longer than actually needed to gradually catch up the leader's behavior. This can be shown by two examples with the same initial conditions below:

Initial positions: $x_{n}(0)=12.81 \mathrm{~m}, x_{n+1}(0)=0 \mathrm{~m}$; Initial speeds: $\dot{x}_{n}(0)=\dot{x}_{n+1}(0)=13.42 \mathrm{~m} / \mathrm{s}$.

It is further assumed that:

- at $t=0,1,2$ and 3 second, the leader's accelerations are respectively $-1.2 \mathrm{~m} / \mathrm{s}^{2},-0.6 \mathrm{~m} / \mathrm{s}^{2}$, $0.6 \mathrm{~m} / \mathrm{s}^{2}$ and $1.2 \mathrm{~m} / \mathrm{s}^{2}$ and the acceleration in each second is uniform;

- the leader accelerates at the rate $a(t)=1.25 \mathrm{~m} / \mathrm{s}^{2}$ at $t=30 \mathrm{~s}$ for $5 \mathrm{~s}$ and then decelerates at $a(t)=-1.25 \mathrm{~m} / \mathrm{s}^{2}$ at $t=100 \mathrm{~s}$ for $5 \mathrm{~s}$;

and the lead vehicle travels at a uniform speed during the rest of the time horizon $[0,150]$. The follower's reaction time $\delta$ is assumed to be $1 \mathrm{~s}$. The two examples distinguish from each other in the following way:

Example 1 (Ex. 1): $m=0, l=1.25$ and $\alpha=9.15$.

Example 2 (Ex. 2): $m=1, l=1.25$ and $\alpha=0.68$.

As shown in Fig. 1a, the lead vehicle decelerates first and then accelerates to the initial speed in the first 4 seconds. Consequently, the distance headway between the two vehicles decreases at first and then increases; they finally reach a steady state. In Ex. 1, the distance headway between the two vehicles recovers its initial value whereas the distance headway in Ex. 2 is $12.44 \mathrm{~m}$ when it gets stabilized. Comparing the two examples suggests that the distance headway from the GM model (1) can be different even when the speeds of the two vehicles are the same at the steady states. The property is determined by the structure of the model. There is no intention to make further discussion here on this issue and the reader of interest may refer to Tordeux et al. (2010), Zhang and Jarrett (1997).

What is concerned here is that the follower's reaction to the leader's acceleration is so slow that the distance headway between them increases (decreases) promptly when the lead vehicle is in a state of accelerating or decelerating constantly. This is demonstrated by 

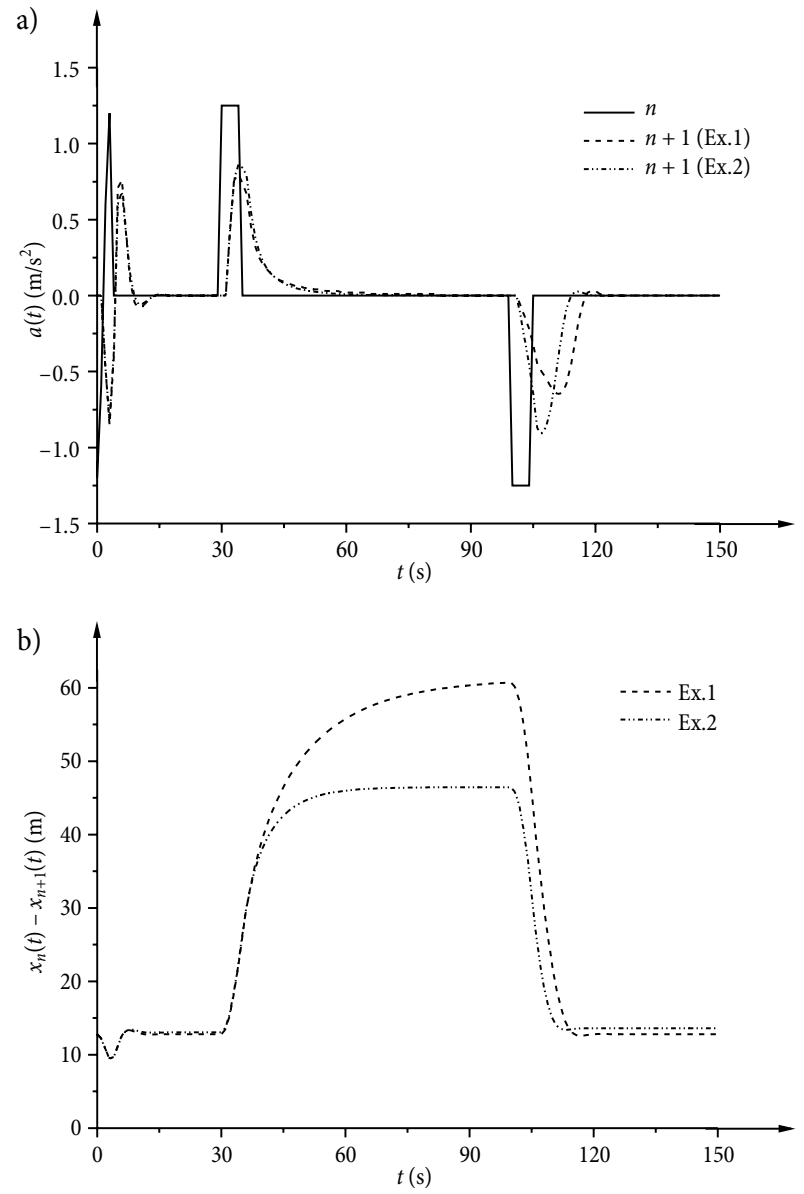

Fig. 1. Solution profiles in Examples 1 and 2: a - acceleration profiles; $b$ - profiles of the distance headways between the lead and following vehicles

the distance headway profiles in Fig. 1b, in particular at $t=30 \mathrm{~s}$ and $t=100 \mathrm{~s}$ when the lead vehicle respectively accelerates and decelerates for $5 \mathrm{~s}$. A more detailed observation follows.

The distance headway between the two vehicles increases quickly when the lead vehicle is accelerating at $a(t)=1.25 \mathrm{~m} / \mathrm{s}^{2}$ for $5 \mathrm{~s}$ at $t=30 \mathrm{~s}$. Until a steady state is reached, the distance headway ceases to increase. It takes longer time to stabilize in Ex. 1 than in Ex. 2; the distance headway in Ex. 1 is much larger than that in Ex. 2. It is arguable that the leader's acceleration has been embedded implicitly into the model by the terms of (the temporal change in) the leader's speed, but it is clear that the follower is not so sensitive to that. As a result, the distance headway increases promptly. In Ex. 1, the distance headway even increases to an unacceptable level. To save space, we put the profiles of the speeds in Fig. 4, together with the speed profiles from the other examples.

Furthermore, the distance headway decreases sharply when the lead vehicle is decelerating at $a(t)=-1.25 \mathrm{~m} / \mathrm{s}^{2}$ for $5 \mathrm{~s}$ at $t=100 \mathrm{~s}$. This may be also due to the fact that model (1) does not incorporate the leader's acceleration directly.
So far, we have identified the three shortcomings of the GM model (1):

- First, it always defines the follower's acceleration as zero whenever the speeds of the lead and following vehicles are equal even when the leader's acceleration is far from zero.

- Secondly, the follower in this model sounds not sensitive enough or in time to the leader's acceleration so that the stable gap between the two vehicles can be too big after the leader continuously accelerates for a while, or the gap decreases too sharply when the lead vehicle decelerates.

- Another concerning property of the model is that the follower's acceleration at time $t+\delta$ is always greater than zero even when the lead vehicle is sharply decelerating at time $t$ as long as the difference in speed between the two vehicles is positive.

In the car-following behavior, a variety of stochastic factors play their roles (e.g. Wagner 2012) but all the above-identified shortcomings of the GM model (1) are not due to the stochasticity. Therefore, it is necessary to overcome them by refining the modelling of the carfollowing behavior. On another hand, we are not saying that the GM model (1) is wrong but want to make it work better by refining it. This paper is proposing a variant of the GM model by embedding the leader's accelerating behavior directly into Equation (1). Section 1 formulates such a model that removes these shortcomings, which can be considered as a variant of the GM model (1). Section 2 numerically compares the varied GM model with the original one. The last section closes the paper up with some concluding remarks.

\section{Model Formulation and Analysis}

\subsection{Modelling}

To investigate the relationship of the follower's acceleration to the leader's acceleration, let us first look at a set of NGSIM data, which was obtained from the I-80 data set of NGSIM for the time interval from 5:00 am to $5: 15 \mathrm{am}$. To exclude the impacts of merging/diverging on the freeway, only the data for those pairs of vehicles traveling along section of the freeway for a significant time interval were extracted and a total of 39 pairs of vehicles are in the dataset. Fig. 2a shows the scatter plot of the follower's acceleration at time $t+\delta$ versus the speed difference at time $t$ between the lead and following vehicles, where the time lag $\delta$ is assumed to be 1 second. Fig. $2 \mathrm{~b}$ depicts the scatter plot of the follower's acceleration at time $t+\delta$ and the leader's acceleration at time $t$. They show that the follower's acceleration is related to the speed difference between the lead and following vehicles and the leader's acceleration. In fact, the coefficients of correlation for (a) and (b) are respectively 0.495 and 0.798 (Fig. 2). 

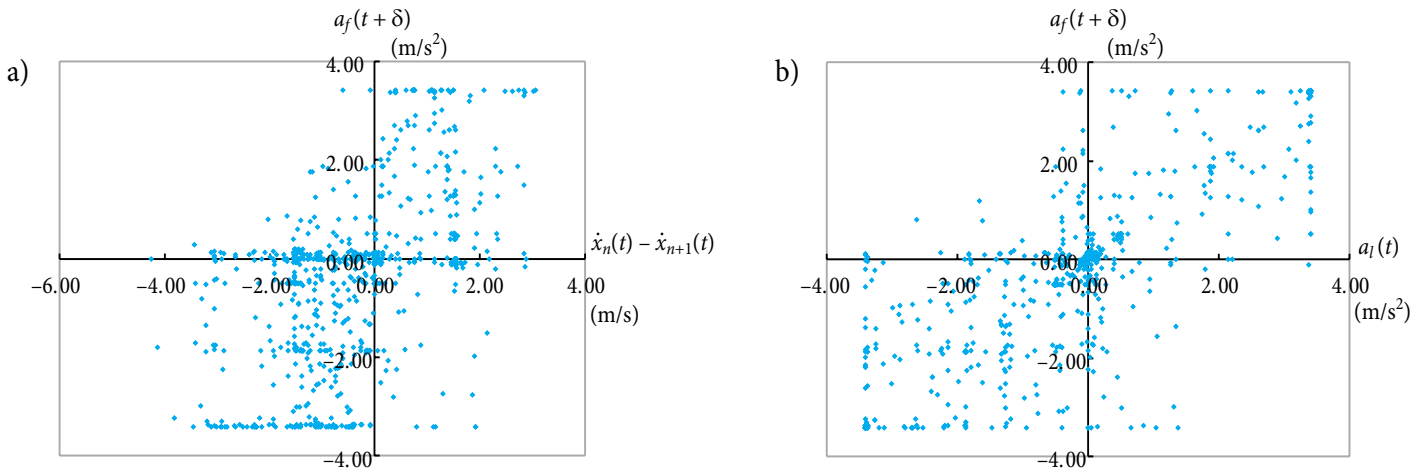

Fig. 2. The relationship of the follower's acceleration $\left(a_{f}(t+\delta)\right)$ to the speed difference from the leader and to the leader's acceleration: $a$ - the follower's acceleration v.s. speed difference from the leader; $b$ - the follower's v.s. leader's acceleration

To overcome the above-identified three shortcomings of the GM model, we base on the analysis results of the NGSIM data above and propose the following new car-following model, which directly adds the leader's acceleration times $\delta$ weighted by a positive coefficient $\beta$ to the 'speed difference' term in model (1), i.e.:

$$
\begin{gathered}
\ddot{x}_{n+1}(t+\delta)=\alpha \frac{\left(\dot{x}_{n+1}(t+\delta)\right)^{m}}{\left(x_{n}(t)-x_{n+1}(t)\right)^{l}} \times \\
\left(\dot{x}_{n}(t)-\dot{x}_{n+1}(t)+\beta \delta \ddot{x}_{n}(t)\right), \beta>0,
\end{gathered}
$$

where: $\beta$ is a parameter that may differ from driver to driver or vary in different traffic conditions (e.g. traffic densities around). All other parameters in model (3) have the same definitions as in model (1), which can be associated with Equation (5) in Sasaki (1959). Kometani and Sasaki (1958) assumes that the spacing between two successive vehicles in queue depends on the velocities of those vehicles, from which it is derived that the speed difference at time $t$ between them is proportional to the acceleration of the following vehicle (Kometani, Sasaki 1958) or a weighted sum of the accelerations of both of them but respectively at $t$ and $t+\delta$ (Sasaki 1959).

Model (3) can be considered as a variant of the original GM model (1). There are two ways to explain the varied GM model. First, the follower's acceleration at $t+\delta$ is proportional to the difference between the leader's approximate velocity at $t+\delta$ (i.e. $\dot{x}_{n}(t)+\beta \delta \ddot{x}_{n}(t)$ ) and the follower's speed at $t$ (i.e. $\dot{x}_{n+1}(t)$ ). If the lead vehicle accelerates at a uniform rate in the time period $[t, t+\delta]$ then $\dot{x}_{n}(t)+\delta \ddot{x}_{n}(t)$ represents the leader's real speed at $t+\delta$.

Secondly, the follower's acceleration at $t+\delta$ is proportional to a weighted sum of the speed difference of the two vehicles at $t$ and the leader's speed change in the following short period of lenght equal to $\delta . \beta=1$ means that the short period is $[t, t+\delta]$.

Integrating Equations (2) and (3) gives:

$$
\ddot{x}_{n+1}(t+\delta)=\lambda\left(\dot{x}_{n}(t)-\dot{x}_{n+1}(t)\right)+\lambda \beta \delta \ddot{x}_{n}(t) \text {. }
$$

As we know, $\lambda$ reflects the follower's sensitivity to the speed difference between the two vehicles. $\lambda \beta \delta$ can be regarded as the follower's sensitivity to the leader's acceleration or $\lambda \beta$ the coefficient of the follower' sensitivity to the speed increment of the lead vehicle within the period $[t, t+\delta]$.

\subsection{Analysis}

This subsection is to check whether model (3) has resolved the above-identified shortcomings of the original GM model (1). First, when the speed difference between the two vehicles is zero but the leader's acceleration is nonzero, the follower's acceleration given by model (3) is proportional to the leader's acceleration, i.e.:

$$
\ddot{x}_{n+1}(t+\delta)=\lambda \beta \delta \ddot{x}_{n}(t), \beta>0,
$$

which is zero only if $\lambda=0 . \lambda=0$ means that $\dot{x}_{n+1}(t+\delta)=0$ holds or that the distance headway between the two vehicles is infinite. The term $\dot{x}_{n+1}(t+\delta)=0$ is an exceptional case, which may not be a situation we tend to model. When the distance headway is infinite, it is unnecessary to use the model to determine the follower's acceleration since the lead vehicle has no influence over the follower at all. Therefore, the new model can ensure that the following vehicle responds to the leader's acceleration in time.

In addition, Equation ( $\left.3^{\prime}\right)$ implies that, since the follower's acceleration equals a weighted sum of the speed difference between the two vehicles and the leader's acceleration, the follower's acceleration may be more likely to become negative when the leader's deceleration is big. If their speed difference is very small then the leader's sharp deceleration will decrease the follower's acceleration quickly. Consequently, the new model can better capture the car-following behavior than the original one when the leader decelerates but its speed is higher than the follower's.

In the new model, the follower's sensitivity to the speed difference between the two vehicles is determined by $\lambda$ while the follower's sensitivity to the leader's acceleration is determined by $\lambda \beta \delta$, see Equation ( $\left.3^{\prime}\right)$. The introduction of $\beta$ gives us one more instrument to capture the follower's sensitivity to the stimuli from the lead vehicle. 
In terms of stability, the new model has the following properties:

- Property 1: The new model is as stable as the original model.

Analysis: A steady state means that the leader's acceleration equals zero, which makes the new model become the original one. Therefore, the new model is as stable as the original one.

- Property 2: At a steady state, the speed-density relationship derived from the new model is the same as that from the original one.

Analysis: At a steady state the leader's acceleration is zero, hence both new and original models become the same. Therefore, their speeddensity relationships are identical.

- Property 3: Under certain conditions, a steady state derived from the new model can ensure that the bigger the speed is the larger the distance headway is.

Analysis: Since at a steady state the speeddensity relationship derived from the new model is the same as that from the original one, the relationship shall ensure that this property holds.

\subsection{A Discussion on Impacts of Each Parameter}

The original model (1) has 3 parameters (i.e. $\alpha, l$ and $m$ ) and the introduction of the new model (3) brings one more parameter $\beta$. The role of $\alpha$ is clear since it linearly amplifies the effects of the leader's behavior on the follower. To observe the effects of the other three parameters, let us assume that the follower's acceleration at $t+\delta$ be a function of these parameters so we can differentiate Equation (3) with respect to (w.r.t.) each of them. In the following analysis, it is assumed that $\alpha$ is a constant.

First, the derivative of the follower's acceleration at $t+\delta$ w.r.t. $l$ is given by:

$$
\begin{aligned}
& \frac{\partial \ddot{x}_{n+1}(t+\delta)}{\partial l}=-\lambda \ln \left(x_{n}(t)-x_{n+1}(t)\right) \times \\
& \left(\dot{x}_{n}(t)-\dot{x}_{n+1}(t)+\beta \delta \ddot{x}_{n}(t)\right) .
\end{aligned}
$$

It can be concluded from formula (2) that $\lambda>0$. It is interesting to see that the sign of the above expression depends on the distance unit used. Suppose that the 'meter' other than 'kilometer' is used and then the distance headway between the two vehicles must be greater than 1 meter since the length of a vehicle plus the safe distance between the two vehicles should be greater than 1 meter at least. Therefore, $\ln \left(x_{n}(t)-x_{n+1}(t)\right)>0$. Note that whether the follower is accelerating or decelerating depends on the sign of $\dot{x}_{n}(t)-\dot{x}_{n+1}(t)+\beta \delta \ddot{x}_{n}(t)$. Integrating this point with Equation (5) shows that the increase in $l$ will result in the decrease in the follower's acceleration when the follower is accelerating and increase the follower's acceleration when the follower is decelerating.

Secondly, the derivative of the follower's acceleration w.r.t. $m$ is:

$$
\begin{aligned}
& \frac{\partial \ddot{x}_{n+1}(t+\delta)}{\partial m}=\lambda \ln \left(\dot{x}_{n+1}(t+\delta)\right) \times \\
& \left(\dot{x}_{n}(t)-\dot{x}_{n+1}(t)+\beta \delta \ddot{x}_{n}(t)\right) .
\end{aligned}
$$

This also shows that the choice of the distance unit affects the sign of the above expression. If $\ln \left(\dot{x}_{n+1}(t+\delta)\right)>0$, the increase in $m$ will result in the increase of the follower's acceleration when the follower is accelerating. However, if $\ln \left(\dot{x}_{n+1}(t+\delta)\right)<0$, the follower's acceleration is a decreasing function of $m$ when the follower is accelerating.

Thirdly, the derivative of the follower's acceleration w.r.t. $\beta$ is given:

$$
\frac{\partial \ddot{x}_{n+1}(t+\delta)}{\partial \beta}=\lambda \delta \ddot{x}_{n}(t) .
$$

This suggests that the follower's acceleration is an increasing (decreasing) function of parameter $\beta$ when the leader is accelerating (decreasing). In other words, the increase in $\beta$ will amplify the effects of the leader's acceleration on the follower's one.

Another thing worth discussing is what factors may affect the parameter value of $\beta$. Let us define $\beta$ in the following form:

$$
\beta=\beta_{0} \frac{\left(x_{n}(t)-x_{n+1}(t)\right)^{l_{0}}}{\left(\frac{\dot{x}_{n}(t)}{v_{e}\left(x_{n}(t)\right)}\right)^{m_{0}}},
$$

where: $m_{0}, l_{0}$ and $\beta_{0}$ are the parameters that need to be calibrated, $m_{0}>0, \beta_{0}>0$ but $l_{0}$ may be positive or negative; $v_{e}\left(x_{n}(t)\right)$ denotes the equilibrium/steady-state speed of vehicle $n$ expected by the follower, which is related to the speed limit, traffic situation, road or weather conditions, etc. The ratio of the speed of vehicle $n$ to the equilibrium speed tends to reflect how much the follower is eager to accelerate: the smaller the ratio is, the more the follower wants to accelerate.

Integrating Equations (2) and (7) gives:

$$
\begin{aligned}
& \lambda \beta=\alpha \beta_{0} \frac{\left(\dot{x}_{n+1}(t+\delta)\right)^{m}}{\left(\frac{\dot{x}_{n}(t)}{v_{e}\left(x_{n}(t)\right)}\right)^{m_{0}}} \times \\
& \frac{1}{\left(x_{n}(t)-x_{n+1}(t)\right)^{l-l_{0}}} .
\end{aligned}
$$

This shows that the sensitivity is proportional to the expected speed of the following vehicle at $t+\delta$ and inversely proportional to the ratio of the speed of vehicle $n$ to the equilibrium speed. Furthermore, if $l_{0}<l$ then the sensitivity is inversely proportional to the distance headway between the two vehicles; if $l_{0}>l$ then the sensitivity is a monotonic increasing function in the distance headway. Therefore, the values of $l_{0}$ and $l$ may depend on the distance headway between the two vehicles.

Now let us investigate how $\beta_{0}, l_{0}$ and $m_{0}$ affect the acceleration of the follower. First, we get:

$$
\frac{\partial \ddot{x}_{n+1}(t+\delta)}{\partial \beta_{0}}=\frac{1}{\beta_{0}} \lambda \beta \delta \ddot{x}_{n}(t) .
$$


This implies that the increase in $\beta_{0}$ will amplify the effect of the leader's acceleration on the follower's one.

The following is the derivative of the follower's acceleration w.r.t. parameter $l_{0}$ :

$$
\frac{\partial \ddot{x}_{n+1}(t+\delta)}{\partial l_{0}}=\lambda \beta \ln \left(x_{n}(t)-x_{n+1}(t)\right) \delta \ddot{x}_{n}(t) .
$$

Note $\lambda \beta \delta>0$. Suppose that the distance unit used here is meter and then $\ln \left[x_{n}(t)-x_{n+1}(t)\right]>0$. Therefore, the increase of $l_{0}$ will result in the increase (decrease) of the follower's acceleration when the leader vehicle is accelerating (decelerating).

The derivative of the follower's acceleration w.r.t. parameter $m_{0}$ is given by:

$$
\frac{\partial \ddot{x}_{n+1}(t+\delta)}{\partial m_{0}}=-\lambda \beta \ln \left(\frac{\dot{x}_{n}(t)}{v_{e}\left(x_{n}(t)\right)}\right) \delta \ddot{x}_{n}(t) .
$$

Suppose that the ratio of the speed of vehicle $n$ to the equilibrium speed greater (less) than one implies that the lead vehicle is decelerating (accelerating) and then the right-hand side of Equation (10) is nonnegative. This suggests that the follower's acceleration is an increasing function in $m_{0}$. When the leader's speed fluctuates around the equilibrium speed, the vehicle normally tends to accelerate or decelerate at a low rate and the follower's acceleration will also fluctuate.

In summary, it is interesting to notice that the direction of the effects of the parameters $l, m$ and $l_{0}$ depends on the distance unit used in the model. The second finding is that the increase in $\beta$ (or $\beta_{0}$ ) will amplify the effect of the leader's acceleration on the follower's one. Thirdly, when the ratio of the speed of vehicle $n$ to the equilibrium speed is close to one or the distance headway between the two vehicles is near one distance unit, the change in the parameters $l, l_{0}$ or $m_{0}$ nearly has no influence over the follower's sensitivity to the stimuli from the lead vehicle.

\section{Numerical Experiments}

\subsection{A Comparison between the New Model and the Original One}

The same initial conditions and time lag of response as in Exs 1 and 2 are used here. Three more examples are set as below:

Example 3 (Ex. 3): $m=m_{0}=0, l=1.25, l_{0}=0.275$, $\alpha=9.15, \beta_{0}=1$. The only difference from Ex. 1 is that the new model is used.

Example 4 (Ex. 4): $m=1, m_{0}=0, l=1.25, l_{0}=0.06$, $\alpha=0.68, \beta_{0}=1$. The only difference from Ex. 2 is that the new model is used.

Exs 3 and 4 do not consider the effects of the equilibrium/stable speed of vehicle $n$ expected by the following vehicle $n+1$ (i.e. $v_{e}\left(x_{n}(t)\right)$ ).

Example 5 (Ex. 5): $m=1, m_{0}=0.025, l=1.25, l_{0}=$ $0.06, \alpha=0.68, \beta_{0}=1$. All parameters and variables in model (3) are considered in this case. It is also assumed that the speed limit of the road of interest is $13.42 \mathrm{~m} / \mathrm{s}$ in the first $30 \mathrm{~s}$ of the time horizon of interest, then becomes $19.67 \mathrm{~m} / \mathrm{s}$ and returns to $13.42 \mathrm{~m} / \mathrm{s}$ at $t=100 \mathrm{~s}$.

Adding the profiles of accelerations and the distance headways from Exs 3-5 to Fig. 1 gives Fig. 3. Note that the profiles of the leader's accelerations are the same in all examples, hence the leader's speed profiles in the 5 examples are identical. Fig. 4 gives the speed profiles of all these examples.

Comparing all profiles in Fig. 3 shows that the follower modeled in the new model (3) is relatively more sensitive to the leader's acceleration than in the original model (1). In addition, it takes model (3) less time than the original one to reach a steady state after the acceleration or deceleration of the lead vehicle. Furthermore, the distance headway between the two vehicles does not increase too much and stays within a reasonable range when model (3) is used. The profiles in Fig. 4 show that the follower's speed can increase quickly up to the leader's speed level when model (3) is used.

The follower's speed or acceleration fluctuates when it nears that of the lead vehicle, as shown in Figs 3 and 4. The fluctuations gradually disappear and then a steady state is reached. However, the original model does not produce this kind of fluctuations. Therefore, the new model seems more capable of capturing the real-life carfollowing behavior.
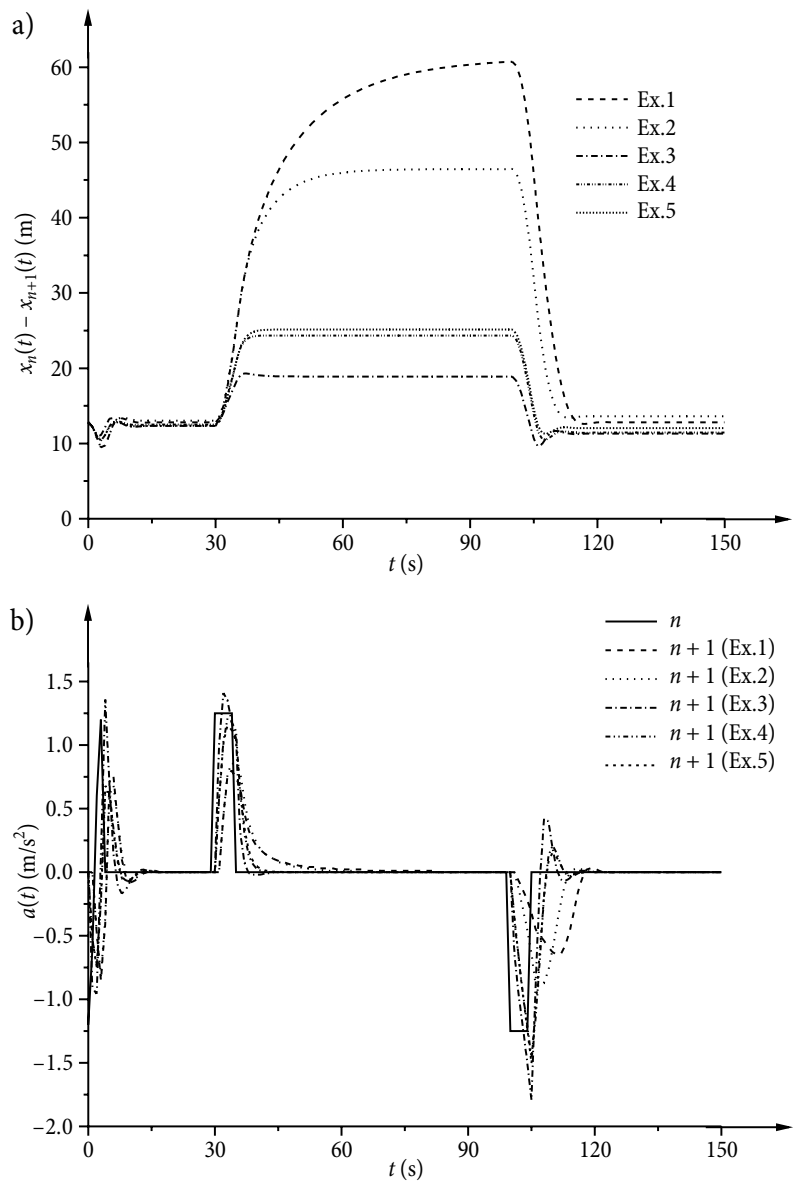

Fig. 3. A comparison of the new model (3) to the original model (1): $\mathrm{a}$ - profiles of the distance headways between the lead and following vehicles; $b$ - acceleration profiles 


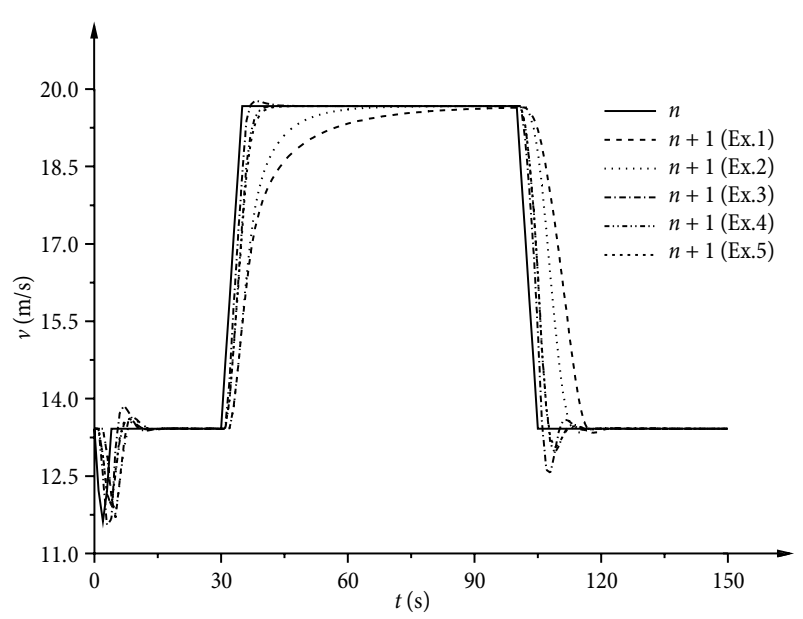

Fig. 4. Speed curves in Exs 1-5

\subsection{The Impacts of the New Parameters}

This subsection intends to observe the impacts of the newly-introduced parameters on the follower's acceleration. The examples in this subsection have the same initial conditions as the previous ones. It is also assumed that the speed limit of the road of interest is $13.42 \mathrm{~m} / \mathrm{s}$ in the first $30 \mathrm{~s}$, then becomes $19.67 \mathrm{~m} / \mathrm{s}$ and comes back to $13.42 \mathrm{~m} / \mathrm{s}$ at $t=100 \mathrm{~s}$.

First, we investigate the impacts of $l_{0}$.

Example 6 (Ex. 6): Suppose $m=1, m_{0}=0.025, l=$ $1.25, \alpha=0.68$ and $\beta_{0}=1$. To observe how the follower's acceleration changes as $l_{0}$ varies, we set $l_{0}$ to three different values, i.e. $0.03,0.06$ and 0.12 .

Equation (7) says that the larger $l_{0}$ is the more sensitive the follower is to the acceleration of the lead vehicle [Note that the distance unit used is meter]. In other words, the larger $l_{0}$ means that it takes the follower less time to adjust his or her own speed to the same level as the leader.

Therefore, the larger $l_{0}$ is, the smaller distance headway in steady state is when the leader is accelerating whereas the larger the distance headway in steady

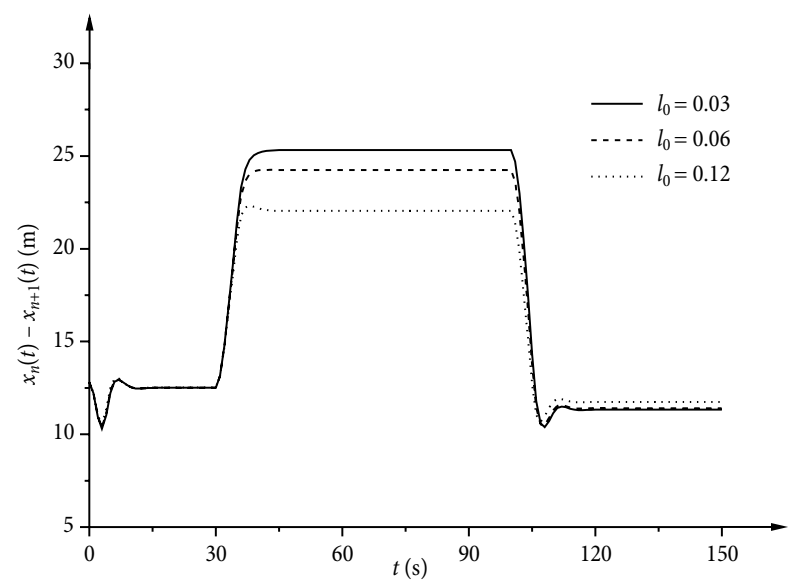

Fig. 5. Profiles of the distance headways between the lead and following vehicles based on the settings in Ex. 6 state is when the lead vehicle is decelerating. This has been demonstrated in Fig. 5.

In Equation $\left(3^{\prime}\right), \delta \ddot{x}_{n}(t)$ is the leader's speed increment within the period from $t$ to $t+\delta$ and has the same unit as the speed difference between the two vehicles. Therefore, $\lambda$ and $\lambda \beta$ are comparable. Fig. 6 gives their profiles, from which we can see that $\beta$ is always no less than one whichever value $\beta$ takes. Therefore, the follower is always more sensitive to the leader's speed increment than the speed difference between the two vehicles. As mentioned before, the original GM model allows the follower to accelerate as long as the speed difference between the lead and follower vehicles is positive, even though the leader is sharply decelerating. The modified model (3) has corrected this problem and enables the follower to respond to the leader's decelerating behavior more quickly.

Equation (8) means that the increase in $l_{0}$ makes $\beta$ or $\lambda \beta$ increase; accordingly, the follower's sensitivity to the leader's acceleration increases. Fig. 6 also clearly shows that the larger distance headway is the less sensitive the follower is to the speed difference between the two vehicles and the leader's acceleration, which is also consistent with the real-life car-following behavior.

Secondly, let us investigate the impacts of $m_{0}$.

Example 7 (Ex. 7): Assume $m=1, l=1.25, l_{0}=$ $0.06, \alpha=0.68, \beta_{0}=1$ and $m_{0}$ takes three values: 0.00125 , 0.025 and 0.5 . Then we will observe the impacts of the value of $m_{0}$ on the distance headway between the two vehicles and the sensitivity coefficients $\lambda$ and $\lambda \beta$.

The profiles of the resulting distance headways from Ex. 7 are depicted in Fig. 7. The figure shows that the larger $m_{0}$ is the more sensitive the follower is to the leader's acceleration. That is, the larger $m_{0}$ is the less time it takes the follower to adjust its speed to the same one of the lead vehicle.

The profiles of $\lambda$ and $\lambda \beta$ derived from Ex. 7 are shown in Fig. 8. It can be seen from Fig. 8 that in the new model the follower is more sensitive to the leader's speed increment than the speed difference between the

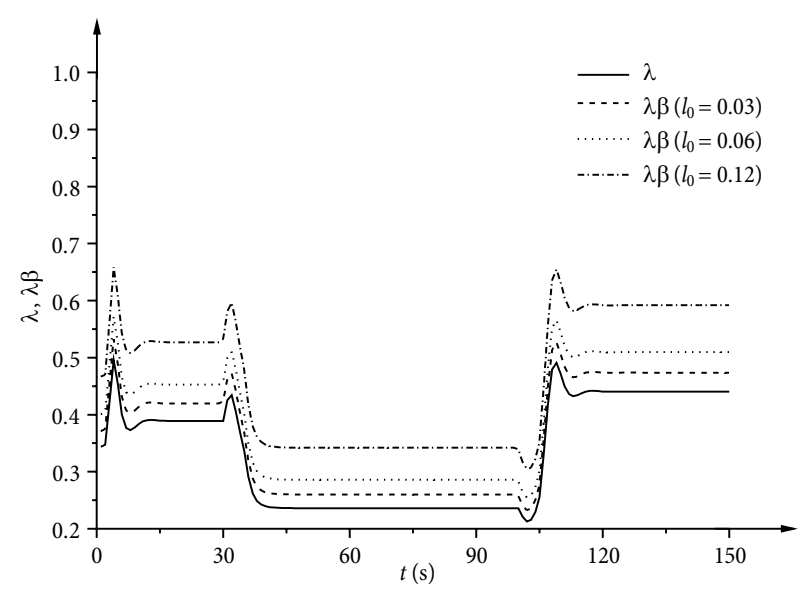

Fig. 6. A comparison between the follower's sensitivity to the speed difference between the two vehicles and that to the leader's acceleration, based on the settings in Ex. 6 
two velocities. It is also clearly revealed in Fig. 8 that the larger the distance headway is the less the follower's sensitivity is to the speed difference and the leader's acceleration, which is similar to what has been found in Ex. 6 .

In Ex. 7, as shown in Fig. 8, when the speed is constant the change in $m_{0}$ has little impact on $\lambda \beta$ since the ratio in the denominator in Equation (7) is normally equal to 1 in this situation. Hence, there are few impacts of $m_{0}$ on the follower's speed and the distance headway between the two vehicles.

Finally, let us consider the impacts of $\beta_{0}$.

Example 8 (Ex. 8): Assume $m=1, m_{0}=0.025, l=$ $1.25, l_{0}=0.065$ and $\alpha=0.68$. To observe the impacts of $\beta_{0}$, we set $\beta_{0}$ to be $0.5,1$, and 1.47 . Then, let us analyse how the profiles of the distance headways between the two vehicles and the sensitivity coefficients $\lambda$ and $\lambda \beta$ change when $\beta_{0}$ moves from one value to another.

The profiles of the distance headways from this example are shown in Fig. 9. The figure shows that the

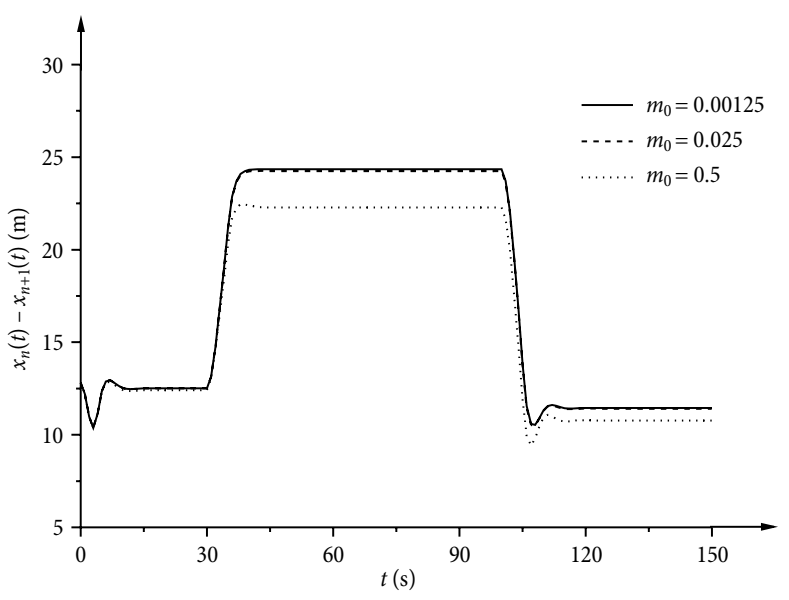

Fig. 7. Profiles of the distance headways between the lead and following vehicles in Ex. 7

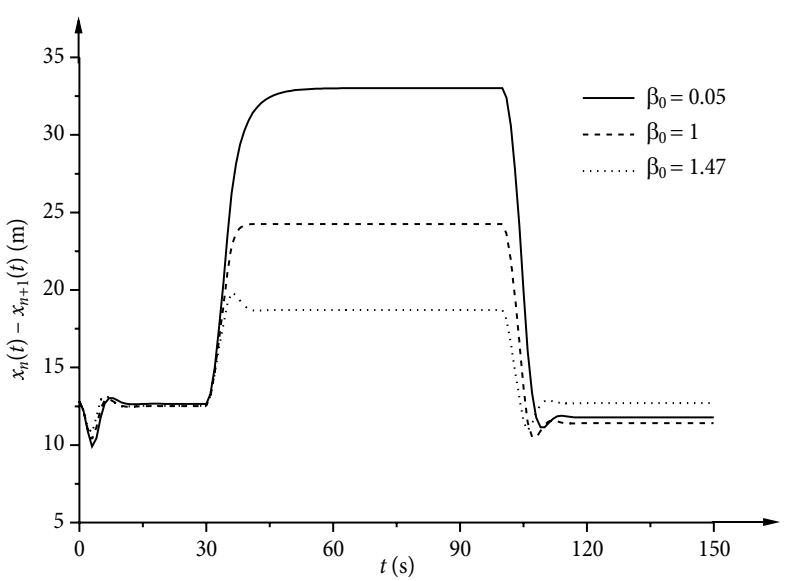

Fig. 9. Profiles of the distance headways between the two vehicles in Ex. 8 larger $\beta_{0}$ is the more sensitive the follower is to the leader's acceleration. In other words, the larger $\beta_{0}$ is the more quickly the follower completes the adjustment at his or her speed to the same one of the lead vehicle.

The profiles of $\lambda$ and $\lambda \beta$ derived from Ex. 7 are shown in Fig. 10. It can be seen from Fig. 10 that, same as in Exs 6 and 7, the larger the distance headway is the less sensitive the follower to the speed difference and the leader's acceleration, which matches the real-life carfollowing behavior well. Fig. 10 also shows that $\beta_{0}$ can take a very flexible role well in the model because it can directly and efficiently adjust the follower's sensitivity to the leader's acceleration.

As shown in Fig. 10, the follower is less sensitive at $\beta_{0}=0.5$ to the acceleration or speed increment within a $\delta$-long period than to the speed difference between the two vehicles, which makes the distance headway become very large for the middle part of the time horizon of interest (see Fig. 9).

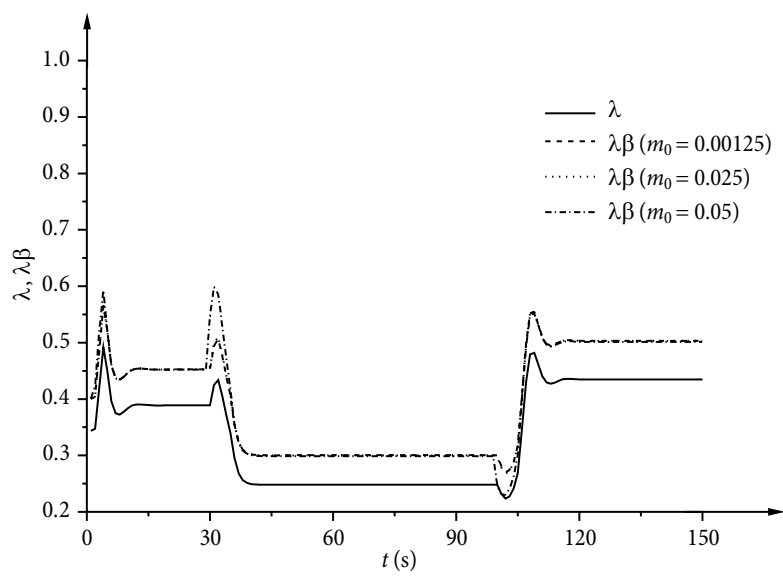

Fig. 8. A comparison between the follower's sensitivity to the speed difference between the two vehicles and that to the leader's acceleration, based on the settings in Ex. 7

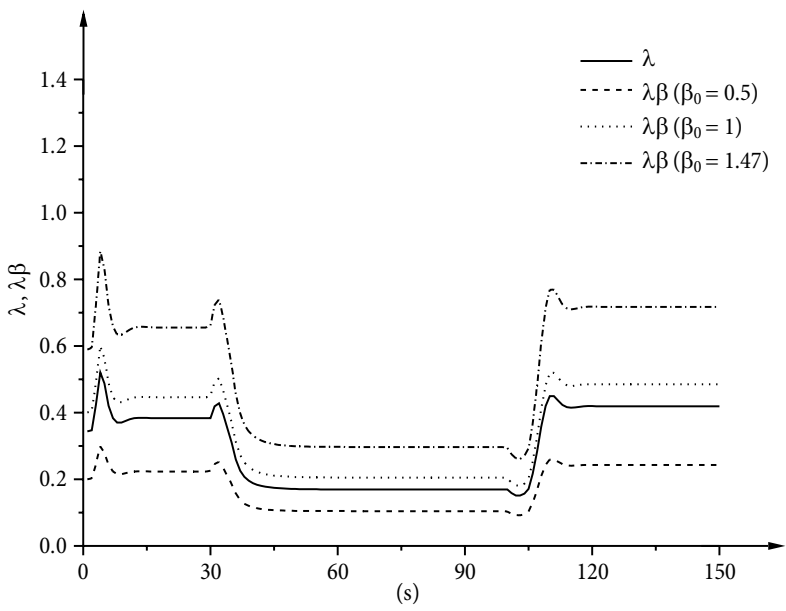

Fig. 10. A comparison between the follower's sensitivity to the speed difference between the two vehicles and that to the leader's acceleration, based on the settings in Ex. 8 


\section{Summary}

Among the various car-following models developed in the past half a century, the family of GM car following model has received the most attention and provided a basic framework to capture the car-following behavior. It is based on the stimulus-response assumption that the following vehicle responds to the relative speed between itself and the leading vehicle. However, some of the empirical findings show that the assumption of GM model is not always true and need some modification. This paper identified three shortcomings of the GM model:

- First, it always yields zero for the follower's acceleration whenever the speed difference between the lead and following vehicles are zero even when the leader is accelerating sharply.

- Secondly, the follower in this model sounds not sensitive enough to the leader's acceleration so that the stable gap between the two vehicles can be too big after the leader continuously accelerates for a while, or the gap decreases too sharply when the lead vehicle decelerates.

- Thirdly, the follower's acceleration at time $t+\delta$ is always greater than zero even when the lead vehicle is sharply decelerating at time $t$ as long as the difference in speed between the two vehicles is positive.

We are not saying that the GM model is wrong but offer a modification to it so it can work better.

To overcome these shortcomings, we have proposed in this paper a new car-following model considering the leader's acceleration directly, in which the follower's acceleration at time $t+\delta$ is proportional to the speed difference between the two vehicles at time $t$ plus the leader's acceleration at time $t$ with a well-designed weight. It has been shown that the new model overcomes the shortcomings of the previous GM model effectively and gives us more instruments to capture the realistic car-following behavior. A series of numerical experiments have been carried out to compare the varied and original GM models and the characteristics of the newly-introduced parameters in the new model are further discussed. However, the calibration of these new parameters, which may not be independent of each other, are remained for the future work.

The examples in this paper show that there may exist different steady states in which the following and lead vehicles have the same speed while they maintain different distance headways. This means that there exists more than one density value associated with the same speed, which is contractive to the argument that a GM model corresponds to one speed-density function. Ge and Sun (2011) has addressed this issue.

In the recent years, the direct effects of acceleration of the lead vehicle have attracted ever-increasing attention in modelling the car-following behavior. Siuhi and Kaseko (2010) showed the need for separate models for acceleration and deceleration responses to address their identified shortcomings of the GM model. Yu et al. (2013) took into account distance headway, relative speed and the leader's acceleration to refine the speed-difference-based car-following models. In addition, as more and more powerful intelligent devices are embedded in transportation infrastructure (e.g. roads) and vehicles, the following vehicle will have more and more ways to perceive the leader's changes in position, speed, acceleration, etc; hence the following drivers can respond to these stimuli more quickly and precisely. Therefore, the research on this issue is doomed to attract more and more researchers' efforts and attention in the near future.

\section{References}

Ahmed, K. I. 1999. Modeling Drivers' Acceleration and Lane Changing Behavior: PhD Thesis. Massachusetts Institute of Technology, USA. 189 p. Available from Internet: http:// dspace.mit.edu/handle/1721.1/9662

Alexiadis, V.; Colyar, J.; Halkias, J.; Hranac, R.; McHale, G. 2004. The next generation simulation program, ITE Journal 74(8): 22-26.

Aw, A.; Klar, A.; Materne, T.; Rascle, M. 2002. Derivation of continuum traffic flow models from microscopic followthe-leader models, SIAM Journal on Applied Mathematics 63(1): 259-278.

http://dx.doi.org/10.1137/S0036139900380955

Brackstone, M.; McDonald, M. 1999. Car-following: a historical review, Transportation Research Part F: Traffic Psychology and Behaviour 2(4): 181-196. http://dx.doi.org/10.1016/S1369-8478(00)00005-X

Ceder, A.; May, A. D. 1976. Further evaluation of single and two regime traffic flow models, Transportation Research Record 567: 1-15.

Chakroborty, P.; Kikuchi, S. 1999. Evaluation of the general motors based car-following models and a proposed fuzzy inference model, Transportation Research Part C: Emerging Technologies 7(4): 209-235.

http://dx.doi.org/10.1016/S0968-090X(99)00020-0

Chandler, R. E.; Herman, R.; Montroll, E. W. 1958. Traffic dynamics: studies in car following, Operations Research 6(2): 165-184. http://dx.doi.org/10.1287/opre.6.2.165

Farhi, N. 2012. Piecewise linear car-following modeling, Transportation Research Part C: Emerging Technologies 25: 100112. http://dx.doi.org/10.1016/j.trc.2012.05.005

Gazis, D. C. 2002. The origins of traffic theory, Operations Research 50(1): 69-77. http://dx.doi.org/10.1287/opre.50.1.69.17776

Gazis, D. C. 1967. Mathematical theory of automobile traffic, Science 157(3786): 273-281. http://dx.doi.org/10.1126/science.157.3786.273

Gazis, D. C.; Herman, R.; Rothery, R. W. 1961. Nonlinear follow-the-leader models of traffic flow, Operations Research 9(4): 545-567. http://dx.doi.org/10.1287/opre.9.4.545

Gazis, D. C.; Herman, R.; Potts, R. B. 1959. Car-following theory of steady-state traffic flow, Operations Research 7(4): 499-505. http://dx.doi.org/10.1287/opre.7.4.499

Ge, Y. E.; Sun, B. 2011. A Car-Following Model with Consistent Separation Distance. Working Paper, Dalian University of Technology and also presented at the 16th International Conference of Hong Kong Society for Transportation Studies, 17-20 December 2011, Hong Kong, China.

Ge, Y. E.; Wu, N.; Sun B. 2011. Coupling pipes car-following model with GM model, Advanced Materials Research 243249: 4392-4401. 
http://dx.doi.org/10.4028/www.scientific.net/AMR.243-249.4392 Greenberg, H. 1959. An analysis of traffic flow, Operations Research 7(1): 79-85. http://dx.doi.org/10.1287/opre.7.1.79

Herman, R.; Montroll, E. W.; Potts, R. B.; Rothery, R. W. 1959. Traffic dynamics: analysis of stability in car following, $O p$ erations Research 7(1): 86-106. http://dx.doi.org/10.1287/opre.7.1.86

Heyes, M. P.; Ashworth, R. 1972. Further research on carfollowing models, Transportation Research 6(3): 287-291. http://dx.doi.org/10.1016/0041-1647(72)90020-2

Kometani, E.; Sasaki, T. 1958. On the stability of traffic flow (Report I), Journal of the Operations Research Society of Japan 2(1): 11-26.

Koutsopoulos, H. N.; Farah, H. 2012. Latent class model for car following behavior, Transportation Research Part B: Methodological 46(5): 563-578.

http://dx.doi.org/10.1016/j.trb.2012.01.001

Lee, G. 1966. A generalization of linear car-following theory, Operations Research 14(4): 595-606. http://dx.doi.org/10.1287/opre.14.4.595

May, A. D.; Keller, H. E. M. 1967. Non-integer car following models, Highway Research Record 199(1): 19-32.

Nagel, K.; Wagner, P.; Woesler, R. 2003. Still flowing: approaches to traffic flow and traffic jam modeling, Operations Research 51(5): 681-710. http://dx.doi.org/10.1287/opre.51.5.681.16755

Ozaki, H. 1993. Reaction and anticipation in the car-following behavior, in Transportation and Traffic Theory: Proceedings of the 12th International Symposium on the Theory of Traffic Flow and Transportation, 21-23 July 1993, Berkeley, California, USA, 349-366.

Sasaki, T. 1959. On the stability of traffic flow (Report II), Journal of the Operations Research Society of Japan 2(2): 60-79.

Sau, J.; Monteil, J.; Billot, R.; El Faouzi, N.-E. 2014. The root locus method: application to linear stability analysis and design of cooperative car-following models, Transportmetrica B: Transport Dynamics 2(1): 60-82. http://dx.doi.org/10.1080/21680566.2014.893416

Simões, M.; Milheiro-Oliveira, P.; Pires da Costa, A. 2010. Modeling and simulation of traffic movements at semiactuated signalized intersections, Journal of Transportation Engineering 136(6): 554-564. http://dx.doi.org/10.1061/(ASCE)TE.1943-5436.0000124

Siuhi, S.; Kaseko, M. S. 2010. Parametric study of stimulusresponse behavior for car-following models, in TRB 89th Annual Meeting Compendium of Papers DVD, 10-14 January 2010, Washington, DC. 19 p. (DVD).

Subramanian, H. 1996. Estimation of Car-Following Models: MSc Thesis. Massachusetts Institute of Technology, USA. 92 p. Available from Internet: http://dspace.mit.edu/handle/1721.1/10660

Tolle, J. E. 1974. Composite car following models, Transportation Research 8(2): 91-96. http://dx.doi.org/10.1016/0041-1647(74)90035-5

Tordeux, A.; Lassarre, S.; Roussignol, M. 2010. An adaptive time gap car-following model, Transportation Research Part B: Methodological 44(8-9): 1115-1131. http://dx.doi.org/10.1016/j.trb.2009.12.018

Treiterer, J.; Myers, J. A. 1974. The hysteresis phenomenon in traffic flow, in Transportation and Traffic Theory: Proceedings of the Sixth International Symposium on Transportation and Traffic Theory, 26-28 August 1974, Sydney, Australia, 13-38.
Wagner, P. 2012. Analyzing fluctuations in car-following, Transportation Research Part B: Methodological 46(10): 1384-1392. http://dx.doi.org/10.1016/j.trb.2012.06.007

Yu, S.; Liu, Q.; Li, X. 2013. Full velocity difference and acceleration model for a car-following theory, Communications in Nonlinear Science and Numerical Simulation 18(5): 12291234. http://dx.doi.org/10.1016/j.cnsns.2012.09.014

Zhang, X.; Jarrett, D. F. 1997. Stability analysis of the classical car-following model, Transportation Research Part B: Methodological 31(6): 441-462.

http://dx.doi.org/10.1016/S0191-2615(97)00006-4 